\title{
Avaliação in vitro do extrato hidroalcoólico (EHA) de alecrim pimenta (Lippia sidoides Cham.) sobre o desenvolvimento de ovos de nematódeos gastrointestinais (Trichostrongylidae)
}

\author{
SOUZA, W.M.A. ${ }^{1 *}$; RAMOS, R.A.N. ${ }^{1}$; ALVES, L.C. ${ }^{2}$; COELHO, M.C.O.C. ${ }^{2}$; MAIA, M.B.S. ${ }^{3}$ \\ ${ }_{1}^{1}$ Aluno do Programa de Pós Graduação em Ciência Veterinária - UFRPE *isabelwagner@uol.com.br \\ ${ }^{2}$ Departamento de Medicina Veterinária - UFRPE, Avenida D. Manoel Medeiros s/n, Dois Irmãos, CEP: 52171 - \\ 900, Recife-Brasil ${ }^{3}$ Departamento de Fisiologia e Farmacologia - UFPE, Avenida Prof. Morais Rego, 1235, \\ Cidade Universitária, CEP: 522670-901, Recife-Brasil
}

\begin{abstract}
RESUMO: Atualmente a fito medicina vem sendo usada no controle de diversas doenças parasitárias particularmente as parasitoses gastrointestinais. Objetivou-se com este estudo avaliar a eficácia do extrato hidroalcoólico (EHA) de Lippia sidoides Cham sobre o desenvolvimento de ovos de nematódeos gastrointestinais. O efeito ovicida foi determinado através de análise probabilística, modelo logístico, utilizados os softwares $R$ versão 2.2.1 e EPI6. Foram obtidos ovos embrionados em fezes de cabras naturalmente infectadas com nematóides Trichostrongylidae e o número de ovos fecais foi determinado usando-se a técnica modificada de McMaster. Cinquenta $\mu \mathrm{L}$ da suspensão contendo 40 ovos foram transferidas a placas de poliestireno e incubadas com 12 concentrações diferentes do EHA sendo avaliada durante 72 horas a temperatura ambiente. Os resultados demonstraram diferentes eficácias para o fito medicamento com menor taxa de desenvolvimento de ovos na concentração de $500 \mathrm{mg} \mathrm{mL}^{-1}$. Conclui-se que o EHA pode ter papel importante sobre o desenvolvimento in vitro de ovos de nematóides gastrintestinais, revelando atividade ovicida.
\end{abstract}

Palavras-chave: ovicida, fito medicina, fitoterápico, caprino

\begin{abstract}
In vitro evaluation of "alecrim pimenta" (Lippia sidoides Cham.) hydroalcoholic extract (HAE) on the development of gastrointestinal nematode (Trichostrongylidae) eggs. Phytomedicine has been currently used in the control of several parasitic diseases, particularly gastrointestinal ones. The aim of this study was to evaluate the efficacy of the hydroalcoholic extract (HAE) from Lippia sidoides Cham. on the development of gastrointestinal nematode eggs. The ovicidal effect was determined through probabilistic analysis, logistic model, by using the programs $R$ version 2.2.1 and Epi Info ${ }^{\mathrm{TM}}$ 6. Embryonated eggs were obtained from feces of goats naturally infected with Trichostrongylidae nematodes and the fecal egg count was determined by using the modified McMaster technique. Fifty microliters of the suspension containing 40 eggs were transferred to polystyrene plates and incubated with 12 different HAE concentrations, and evaluations were performed during $72 \mathrm{~h}$ at room temperature. The results demonstrated different efficacy of extracts, with lower egg development rate at $500 \mathrm{mg} \mathrm{mL}^{-1}$. In conclusion, HAE may play an important role on the in vitro development of gastrointestinal nematode eggs, indicating ovicidal activity.
\end{abstract}

Key words: ovicide, phytomedicine, phytotherapeutics, goat

\section{INTRODUÇÃO}

A Lippia sidoides (Verbanaceae) ou alecrim pimenta é planta bastante usada como fitoterápico no nordeste brasileiro em função da ação anti-séptica devido aos altos teores de timol e carvacrol (Matos \&
Oliveira, 1998).

O uso de fitoterápicos em medicina veterinária tem crescido muito nos últimos anos, e várias plantas com atividade anti-helmíntica tem sido

Recebido para publicação em 17/02/2009

Aceito para publicação em 18/04/2010

Rev. Bras. PI. Med., Botucatu, v.12, n.3, p.278-281, 2010. 
testadas (Vieira \& Cavalcante, 1999; Assis, 2000; Pessoa, 2001; Camurca-Vasconcelos et al., 2008).

Nos países em desenvolvimento os parasitos gastrointestinais dos ruminantes representam um dos fatores mais importantes na diminuição da produtividade dos rebanhos (Waller et al., 1996), sendo o controle realizado através de tratamento essencialmente químico, onde as drogas utilizadas deixam resíduos nos animais ocasionando um problema para de saúde pública (Padilha et al., 2000).

Segundo Vieira \& Cavalcante (1999), a utilização destes produtos é um método alternativo viável que pode reduzir as dosificações antihelmínticas, aumentando a sobrevida dos produtos químicos disponíveis no mercado.

Este trabalho objetivou avaliar a ação ovicida in vitro do extrato hidroalcoólico (EHA) de Lippia sidoides Cham. sobre o desenvolvimento de ovos de nematódeos gastrointestinais (Trichostrongylidae).

\section{MATERIAL E MÉTODO}

\section{Obtenção do extrato (EHA)}

Foram utilizadas folhas de L. sidoides Cham. provenientes da região do Semi-árido pernambucano (825'00"Sul e 3703'15"Oeste) e transportadas ao laboratório de produtos bioativos de Departamento de Fisiologia e Farmacologia, da Faculdade de Farmácia da Universidade Federal de Pernambuco onde o extrato vegetal foi obtido. Inicialmente as folhas foram trituradas e desidratadas em estufa durante 1 hora a $50^{\circ} \mathrm{C}$. Em seguida foi adicionado Etanol/ $\mathrm{H} 2 \mathrm{O} 70^{\circ} \mathrm{GL}$ (1:1), e procedida a homogeneização e incubação em banho-maria a $70^{\circ} \mathrm{C}$ por $1 \mathrm{~h}$ e $30 \mathrm{~min}$. Após este período, foi realizada a filtração em funil com algodão e o extrato obtido foi concentrado em rota evaporador (com pressão reduzida à temperatura de $50^{\circ} \mathrm{C} 90 \mathrm{rpm}$ ), A L. sidoides Cham. foi identificada e tombada no Herbário IPA-PE sob no 82505 pela botânica Olívia Cano, funcionária da mesma instituição.

\section{Obtenção dos ovos de nematódeos Gastrointestinais}

Foram coletadas 10 grama de fezes diretamente da ampola retal de 20 animais da espécie caprina criados em sistema semi-intensivo no município de Jaboatão dos Guararapes (8ำ10'00" Sul e 350'00" Oeste), Pernambuco. As fezes foram acondicionadas em sacos plásticos e encaminhadas sob temperatura refrigerada ao Laboratório de Doenças Parasitárias dos Animais Domésticos da Universidade Federal Rural de Pernambuco.

Todas as amostras fecais foram submetidas ao exame parasitológico através da técnica de McMaster (OPG) Gordon \& Whitlock (1929) para padronização do número de ovos a serem utilizados. Apenas as amostras com OPG superior a 3000 ovos por grama de fezes foram utilizadas como fonte de ovos para avaliação do efeito ovicida do fitoterápico.

Um pool de fezes foi preparado, obtendose apenas uma única amostra de $10 \mathrm{~g}$, sendo adicionada solução saturada de açúcar em volume de $60 \mathrm{~mL}$, e procedida a filtração em tamiz de 250 $\eta \mathrm{m}$ e $180 \mathrm{\eta m}$. Em seguida alíquotas de $50 \mu \mathrm{L}$ foi retirada para contagem e padronização da quantidade de ovos presentes na solução. Após a determinação média da concentração dos ovos em $50 \mu \mathrm{L}$ da solução foi retirado igual volume e aplicado em placas de poliestireno com 96 cavidades de fundo chato para a realização dos testes.

\section{Determinação da atividade ovicida}

Para realização dos testes, diferentes concentrações do EHA L. sidoides $(\mathbf{T})$ tratamentos ( $\mathbf{T}$ 1 controle água destilada, T2 1,0 $\mathrm{mg} \mathrm{mL}^{-1}$, T3 2,0 $\mathrm{mg} \mathrm{mL}^{-1}$, T4 5,0 $\mathrm{mg} \mathrm{mL}^{-1}$, T5 $10 \mathrm{mg} \mathrm{mL}^{-1}$, T6 $20 \mathrm{mg} \mathrm{mL}^{-1}$, T7 $\mathrm{mg} \mathrm{mL}^{-1}$, T8 $100 \mathrm{mg} \mathrm{mL}^{-1}$, T9 mg $\mathrm{mL}^{-1}, \mathrm{~T} 10250 \mathrm{mg} \mathrm{mL}^{-1}$ e T11 $500 \mathrm{mg} \mathrm{mL}^{-1}$ do extrato vegetal e T12 febendazole $33 \mathrm{mg} \mathrm{mL}^{-1}$ ). Foram adicionadas aos poços contendo $50 \mu \mathrm{L}$ de ovos de nematódeos gastrointestinais, e as placas foram incubadas a temperatura ambiente de $26^{\circ} \mathrm{C}$, sendo avaliado o desenvolvimento de ovos de nematódeos gastrointestinais (Trichostrongylidae) nos tempos de 1, 3, 6, 12, 24, 48 e 72 hora, em microscopia ótica de 100x, observando a presença de blástula, mórula e ovo larvado em todos os tempos.

O efeito in vitro do EHA sobre o desenvolvimento dos ovos e larvas foi analisado utilizando-se o teste de eclodibilidade para determinação de eficácia anti-helmíntica; proposto por Batista et al. (2002). Foram feitas três repetições do experimento constituindo assim um total de 252 unidades experimentais.

Para a determinação da eficácia antihelmíntica foi utilizado o teste de eclodibilidade proposto por Colps et al. (1992).

Para análise estatística foi utilizado modelo logístico através dos softwares $R$ versão 2.2.1 e EPI6. Todas as decisões foram tiradas ao nível de significância de 5\%.

\section{RESULTADO E DISCUSSÃO}

O desenvolvimento de ovos de nematódeos gastrintestinais foi observado após 12 horas de incubação (Figura 1).

A ausência de evolução dos ovos nas primeiras horas do experimento se deve principalmente a própria evolução fisiológica do parasito, que

Rev. Bras. Pl. Med., Botucatu, v.12, n.3, p.278-281, 2010. 


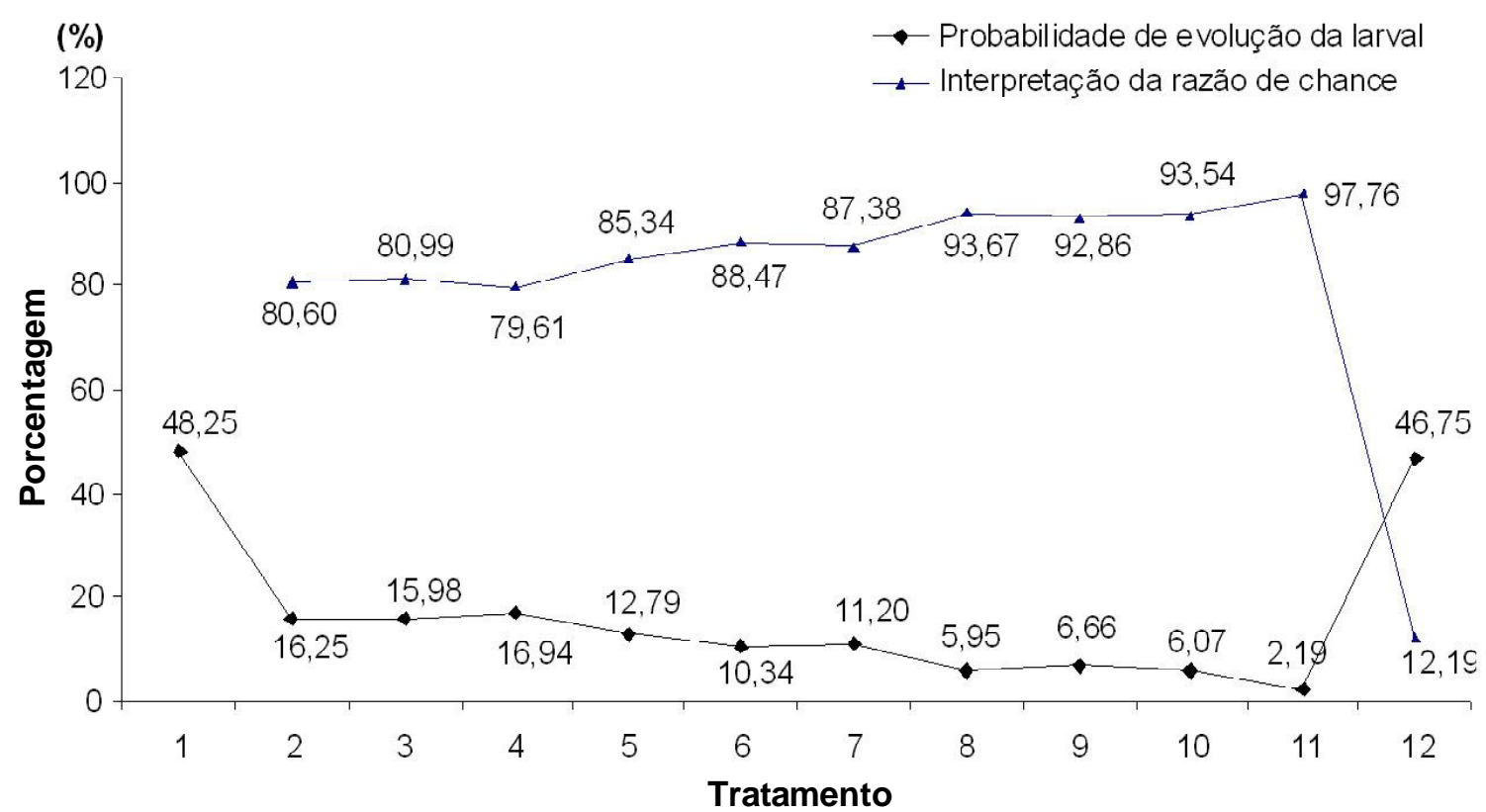

FIGURA 1. Probabilidade de evolução larval após tratamento com EHA de L. sidoides nas diferentes concentrações.

segundo Furtado (2006), só ocorre após algumas horas de presença no meio ambiente.

No grupo controle água T1 a probabilidade de ocorrer evolução de um ovo quando tratado com esta solução foi de aproximadamente 0,48 ou $48 \%$ ocorrendo neste percentual evolução total da larva. Os grupos tratamentos apresentaram-se com probabilidades praticamente relativamente baixas de evolução e possuem cerca de $80 \%$ vezes chance a menos de evolução.

Por outro lado, foi observada equivalência de probabilidade sem diferenças significativas entre os grupos T8, T9 e T10 com resultados na ordem de 0,06 ou $6 \%$ de chance de evolução do ovo tratado e cerca de $94 \%$ vezes chance a menos de evolução que o tratamento $\mathrm{T} 1$.

O grupo Tratamento T11 apresentou probabilidade de ocorrer evolução de um ovo quando tratado em $500 \mathrm{mg} \mathrm{mL}^{-1}$ de aproximadamente 0,02 , melhor desempenho probabilístico, não permitindo o total desenvolvimento larval, superando todos os grupos testados, sendo também superior ao controle positivo T12.

Os dados observados neste estudo discordam de Furtado (2006) ao relatar que estes ovos ao chegarem ao meio externo conjuntamente com as fezes, e em temperatura favorável evoluem para um ovo larvado em primeiro estagio (L1) e após algumas horas os ovos eclodem e liberam as larvas rabditóides. $O$ que não ocorreu neste experimento durante os períodos testados após a adição do extrato da $L$. sidoides.

Os resultados encontrados revelam um potencial ovicida in vitro desenvolvido pelo EHA de
Lippia sidoides Cham sobre nematódeo gastrointestinal de caprinos, corroborando com os dados de Bevillaqua et al. (2005), que avaliaram a atividade ovicida do óleo esssencial de Lippia sidoides encontrando $100 \%$ de ação positiva sobre os mesmos parasitos nas contrações de 2,1 e $0,5 \%$. Essa atividade também foi confirmada por Pessoa (2001), que encontrou nos óleos essenciais de Chenopodium ambrosioides, Ocimum gratissimum, L. sidoides e Cróton zehntneri, ação sobre $H$. contortus.

Em relação ao momento da observação, aqui foram realizadas leituras nos tempos de $1,3,6,12$, 24, 48 e 72 horas, discordando de Furtado (2006) interrompendo-se a evolução, no momento da leitura, com a adição de lugol a 1\%. Com isto se buscou observar que o extrato embora demonstrasse ser probabilisticamente efetivo em 24 horas, permitisse uma evolução nas 48 horas restantes, ou seja, não inibindo a evolução e sim retardando o desenvolvimento dos ovos, corroborando assim com Batista et al. (2002), que observaram que o retardo da eclosão de ovos e a atuação do extrato sobre larvas podem resultar na eliminação de ovos inférteis.

No grupo controle positivo T12 a probabilidade de ocorrer evolução de um ovo quando tratado em febendazole foi de aproximadamente 0,47. Além disso, possui cerca de $2 \%$ vezes chances a menos de evolução que o tratamento água permitindo em alguns casos a evolução embrionária.

As análises dos testes nos tempos referidos não apresentaram diferenças significativas, nos revelando que a variação 
probabilística se dava apenas nas mudanças das concentrações usadas no experimento.

Após as 72 horas de análise dos ovos de nematódeos gastrointestinais de caprinos não foi observado nos grupos testados com o EHA de Lippia sidoides e nas diversas concentrações nenhuma eclosão de ovos e consequentemente presença de Larvas L1 na solução. O mesmo não ocorreu com o grupo controle água, que ao final do mesmo período além de elevado número de ovos larvados apresentou 29 larvas rabditóides no meio aquoso.

Estudos in vivo realizados por CamurçaVasconcelos et al. (2008), em pequenos ruminantes no controle de parasitos gastrintestinais com 200 $\mu \mathrm{g} \mathrm{kg}^{-1}$ lactona macrocíclica (ivernctina) e $283 \mathrm{mg} \mathrm{kg}^{-1}$ do óleo essencial de Lippia sidoides, revelaram uma eficácia equivalente entre os produtos testados na ordem de 40.2 e $45.9 \%$ nos primeiros sete dias de estudo, 39.6 e $54 \%$ no décimo quarto respectivamente, corroborando com os dados encontrados para comprovação de possível atividade anti-helmíntica da L. sidoides .

\section{CONCLUSÃO}

O EHA de Lippia sidoides Cham na concentração de $500 \mathrm{mg} \mathrm{mL}^{-1}$ obteve ação ovicida in vitro ao reduzir a $2 \%$ a probabilidade de evolução embrionária dos ovos de nematódeos gastrointestinais de família Trichostrongylidae.

\section{REFERÊNCIA}

ASSIS, L.M. Atividade antihelmíntica in vitro de extratos de Spigelia anthelmia sobre Haemonchus controtus. 2000. 44p. Dissertação (Mestrado em Ciência Veterinária) - Faculdade de Medicina Veterinária, UECE, Fortaleza.

BATISTA, L.M.; BEVILAQUA, C.M.L.; MORAES, S.M. Efeito ovicida e larvicida dos extratos de Spigelia anthelmia e
Monocardia charantia sobre nematodeo Haemonchos contortus. Revista Brasileira de Farmacognosia, v.12, n.2, p.81-2, 2002. Disponível em: <http//www. sfcgnosia.org.br/ admin/pages/revista/ar>. Acesso em: 15 mai. 2007

BEVILAQUA, C.M.L. et al. Ovicidal and larvicidal activity of Lippia sidoides and Ocimum gratissimum essencial oils against Haemonchus contortus. Proceedings oft the 20th International conference of the World Association for the Advancement of Veterinary Parasitology, Christchurch.

New Zealand Veterinary Journal, v.1, p.78-9, 2005. Disponível em: <http//www sci quest.org.nz.pdf>. Acesso em: 26 set. 2006.

CAMURCA-VASCONCELOS, A.L.F. et al. Anthelmintic activity of Lippia sidoides essential oil on sheep gastrointestinal nematodes. Veterinary Parasitology, v.154, n1-2, p.167-70, 2008. Disponível em: <http//www. Sciencedirect.com/sience?pdf>. Acesso em: 14 abr. 2009. COLPS, G.C. et al. Methods for the detection of anthelmintic resistance in nematodes of veterinary importance. Veterinary Parasitology, v.44, p.35-44, 1992.

FURTADO. K.S. et al. Efeito de Carica paya L. e Musa paradisiaca Linn. sobre o desenvolvimento de ovos de nematódeos gastrintestinais de ovinos. Arquivo Instituto Biológico, v.72, n.2, p.191-7, 2006.

GORDON, H.M.C.L.; WHITLOCK, A.V. A new technique for counting nematode eggs in sheep feces. Journal Council Scientific Industry Research, v.12, p.50-2, 1939. MATOS, F.J.A.; OLIVEIRA, F. Farmácias vivas - plantas medicinais com atividade antifúngica. Revista Brasileira de Farmácia, v.79, p.84-7, 1998.

PADILHA, T. et al. Genética: a nova arma no controle de doenças. Balde Branco, v.36, n.229, p.58-62, 2000.

PESSOA, L.M. Atividade ovicida in vitro de plantas medicinais contra Haemonchos contortus. 2001. 68p. Dissertação (Mestrado em Parasitologia Veterinária) Departamento de Medicina Veterinária, UFCG, Campina Grande.

VIEIRA, L.S.; CAVALCANTE, A.C.R. Resistência antihelmíntica em rebanhos caprinos no Estado do Ceará Pesquisa Veterinária Brasileira, v.19, n.3/4, p.99-103, 1999.

WALLER, P.J. et al. The prevalence of anthelmintic resistance in nematode parasites of sheep in Southern Latin America: general overview. Veterinary Parasiltology, v.62, p.181-7, 1996. 